\title{
Diffusion of Colloidal Rods in Corrugated Channels
}

\author{
Xiang Yang, ${ }^{1}$ Qian Zhu, ${ }^{1}$ Chang Liu, ${ }^{1}$ Wei Wang, ${ }^{2}$ Yunyun \\ $\mathrm{Li}^{3}{ }^{3}$ Fabio Marchesoni, ${ }^{3,4}$ Peter Hänggi, ${ }^{5,6}$ and H. P. Zhang ${ }^{1,7}$ \\ ${ }^{1}$ School of Physics and Astronomy and Institute of Natural Sciences, \\ Shanghai Jiao Tong University, Shanghai, China \\ ${ }^{2}$ School of Material Science and Engineering, \\ Harbin Institute of Technology, Shenzhen Graduate School, Shenzhen, China \\ ${ }^{3}$ Center for Phononics and Thermal Energy Science, \\ School of Physics Science and Engineering, \\ Tongji University, Shanghai, China \\ ${ }^{4}$ Dipartimento di Fisica, Università di Camerino, I-62032 Camerino, Italy \\ ${ }^{5}$ Institut für Physik, Universität Augsburg, D-86135 Augsburg, Germany \\ ${ }^{6}$ Nanosystems Initiative Munich, Schellingstrasse 4, D-80799 München, Germany \\ ${ }^{7}$ Collaborative Innovation Center of Advanced Microstructures, Nanjing, China
}

(Dated: February 11, 2019)

\begin{abstract}
In many natural and artificial devices diffusive transport takes place in confined geometries with corrugated boundaries. Such boundaries cause both entropic and hydrodynamic effects, which have been studied only for the case of spherical particles. Here we experimentally investigate diffusion of particles of elongated shape confined into a corrugated quasi-two-dimensional channel. Elongated shape causes complex excluded-volume interactions between particle and channel walls which reduce the accessible configuration space and lead to novel entropic free energy effects. The extra rotational degree of freedom also gives rise to a complex diffusivity matrix that depends on both the particle location and its orientation. We further show how to extend the standard Fick-Jacobs theory to incorporate combined hydrodynamic and entropic effects, so as, for instance, to accurately predict experimentally measured mean first passage times along the channel. Our approach can be used as a generic method to describe translational diffusion of anisotropic particles in corrugated channels.
\end{abstract}


Diffusive transport through micro-structures such as occurring in porous media [1, 2], micro/nano-fluidic channels [3-7] and living tissues [8, 9], is ubiquitous and attracts evergrowing attention from physicists [10, 11], mathematicians [12], engineers [1], and biologists [8, 9, 13]. A common feature of these systems are confining boundaries of irregular shapes. Spatial confinement can fundamentally change equilibrium and dynamical properties of a system by both limiting the configuration space accessible to its diffusing components [10] and increasing the hydrodynamic drag [14] on them.

An archetypal model to study confinement effects consists of a spherical particle diffusing in a corrugated narrow channel, which mimics directed ionic channels [15], zeolites [16], and nanopores [17]. In this context, Jacobs [18] and Zwanzig [19] proposed a theoretical formulation to account for the entropic effects stemming from constrained transverse diffusion. Focusing on the transport (channel) direction, they assumed that the transverse degrees of freedom (d.o.f's) equilibrate sufficiently fast and can, therefore, be eliminated adiabatically by means of an approximate projection scheme. In first order, they derived a reduced diffusion equation in the channel direction, known as the Fick-Jacobs (FJ) equation. Numerical investigations [11, 20 23] demonstrated that the FJ equation provides a useful tool to accurately estimate the entropic effects for confined pointlike particles. However, our recent experiments [5] evidentiated that hydrodynamic effects for finite size particles cannot be disregarded if the channel and particle dimensions grow comparable. In order to incorporate such hydrodynamic corrections, the FJ equation must then be amended in terms of the experimentally measured particle diffusivity.

Previous studies on confined diffusion focused mostly on spherical particles, for which only the translational d.o.f's were considered. However, particles in practical applications appear inherently more complex in exhibiting anisotropic shape and possessing additional degrees of freedom other than translational. For example, anisotropic particles, such as colloids [24 28], artificial and biological filaments [29, 30], DNA strands [31, 32] and microswimmers [33, 34], exhibit complex coupling between rotation and translation, even in the absence of geometric constraints. How can complex shape and additional d.o.f's such as rotation alter the current picture of confined diffusion? Here, we address this open question and study how a colloidal rod diffuses in a quasi-two-dimensional (2D) corrugated channel [35]. Our experiments reveal that the interplay of channel's spatial modulation, rod's shape and rotational dynamics causes substantial hydrodynamic and entropic effects. We succeed to 

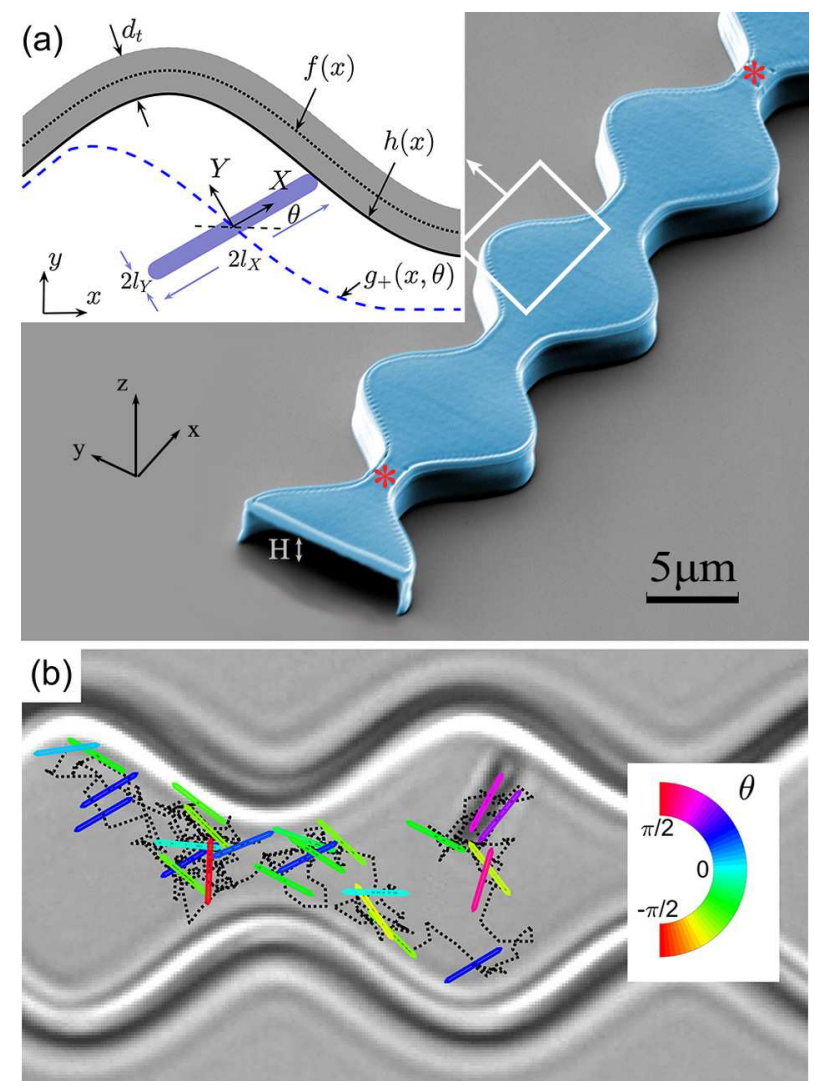

Figure 1. (a) Electron scanning image of a thin channel $(H=1.0 \mu \mathrm{m}, \alpha=7 / 8)$. Narrow openings at the two ends are marked by red asterisks. The inset illustrates a section of the channel with laser-scanning contour, $f(x)$, wall inner boundary, $h(x)$, and upper effective boundary, $g_{+}(x, \theta)$, delimiting the region accessible to the center of a rod with a given tilting angle, $\theta$. Rod's length and width and wall thickness are denoted respectively by $2 l_{X}, 2 l_{Y}$ and $d_{t}$. The coordinates $x, y, z$ and $X, Y$ refer respectively to the laboratory and body frames. (b) Sample of time discretized trajectory (dotted line) for a rod with $l_{X}=1.5 \mu \mathrm{m}$ in a tall channel $(H=2.0 \mu \mathrm{m}, \alpha=1)$; the rod's orientation at different times is also reported according to the depicted color-code.

extend the standard FJ theory to incorporate both effects; the resulting theory accurately predicts the experimentally measured mean first-passage times (MFPT's) associated with rod translation along the channel.

Experimental setup. Our channels were fabricated on a coverslip by means of a twophoton direct laser writing system, which solidifies polymers according to a preassigned channel profile, $f(x)$, with a submicron resolution [5]. As depicted in Fig.1 (a), the quasi-2D channel has a uniform height (denoted by $H$ ). In the central region, the periodically curved 
lateral walls form cells of length $L$ with inner boundaries a distance $y= \pm h(x)$ away from the channel's axis. The preassigned profile $f(x)$ is given the form of a cosine, which tapers off to a constant in correspondence with the cell connecting ducts, or necks, that is

$$
f(x)=\left\{\begin{array}{c}
\frac{1}{2}\left(f_{w}+f_{n}\right)+\frac{1}{2}\left(f_{w}-f_{n}\right) \cos \left(\frac{2 \pi x}{\alpha L}\right),|x|<\frac{\alpha L}{2} \\
f_{n}, \frac{\alpha L}{2} \leq|x|<\frac{1}{2} L
\end{array} .\right.
$$

The minimum (maximum) half-width of $f(x)$ is denoted by $f_{n(w)}$, respectively, whereas $(1-\alpha) L$ is the length of the neck. Due to the lateral wall thickness $d_{t}=0.8 \mu \mathrm{m}$ [see inset of Fig. 1(a)], $f(x)$ and $h(x)$ are separated by a distance $d_{t} / 2$, so that $f_{n(w)}=h_{n(w)}+d_{t} / 2$. We changed $f_{n}$ continuously for fixed $L=12 \mu \mathrm{m}$ and $f_{w}=4.6 \mu \mathrm{m}$, while for the remaining channel parameters we considered two typical geometries: tall channels $(H=2.0 \mu \mathrm{m}, \alpha=1)$ and thin channels $(H=1.0 \mu \mathrm{m}, \alpha=7 / 8)$.

After fabrication, channels were immersed in water with suspended iron-plated gold rods of width $2 l_{Y}=0.3 \mu \mathrm{m}$ and length $2 l_{X}$, which varies in the range 1.6-3.2 $\mu \mathrm{m}$. Using a magnet, we dragged a rod into the channel through a narrow entrance, which creates insurmountable entropic barriers to prevent the rod from exiting the channel. The rod's motion in such quasi-2D channel was recorded through a microscope at 30 frames per second for up to $20 \mathrm{~h}$ [5]. We tracked rod trajectories in the imaging plane and extracted its center coordinates, $(x, y)$, and tilting angle, $\theta$, by standard particle-tracking algorithms. We detected no sizeable rod dynamics in the out-of-plane direction, see Movie S1.mp4 in Supplemental Material [36].

A typical rod trajectory is displayed in Fig. 1(b). The channel boundaries limit the space accessible to the rod and such a limiting effect depends on the rod's orientation: the rod gets closer to the boundary if it is aligned tangent to the walls. To quantify this orientation dependent effect, we distributed the recorded rod's center coordinates, $(x, y)$, for a given orientation, $\theta$, into small bins $(0.26 \mu \mathrm{m} \times 0.2 \mu \mathrm{m})$ and counted how many times the rod's center was to be found in each bin. The resulting rod center distributions for three values of $\theta$ are plotted in Fig. 2(a). Nearly uniform distributions demonstrate that the rod diffuses in a flat energy landscape, whereas sharp drops of the distributions near the boundaries mark the edge of the accessible space, consistently with $y=g_{ \pm}(x, \theta)$ computed from the excluded-volume considerations [see Fig. 2(a)]. The channel boundaries also affect the rod's orientation. For instance, when the rod is relatively long, namely for $h_{n}<l_{X}$, then it tends to orient itself parallel to the channel direction inside the neck region, as illustrated in the 

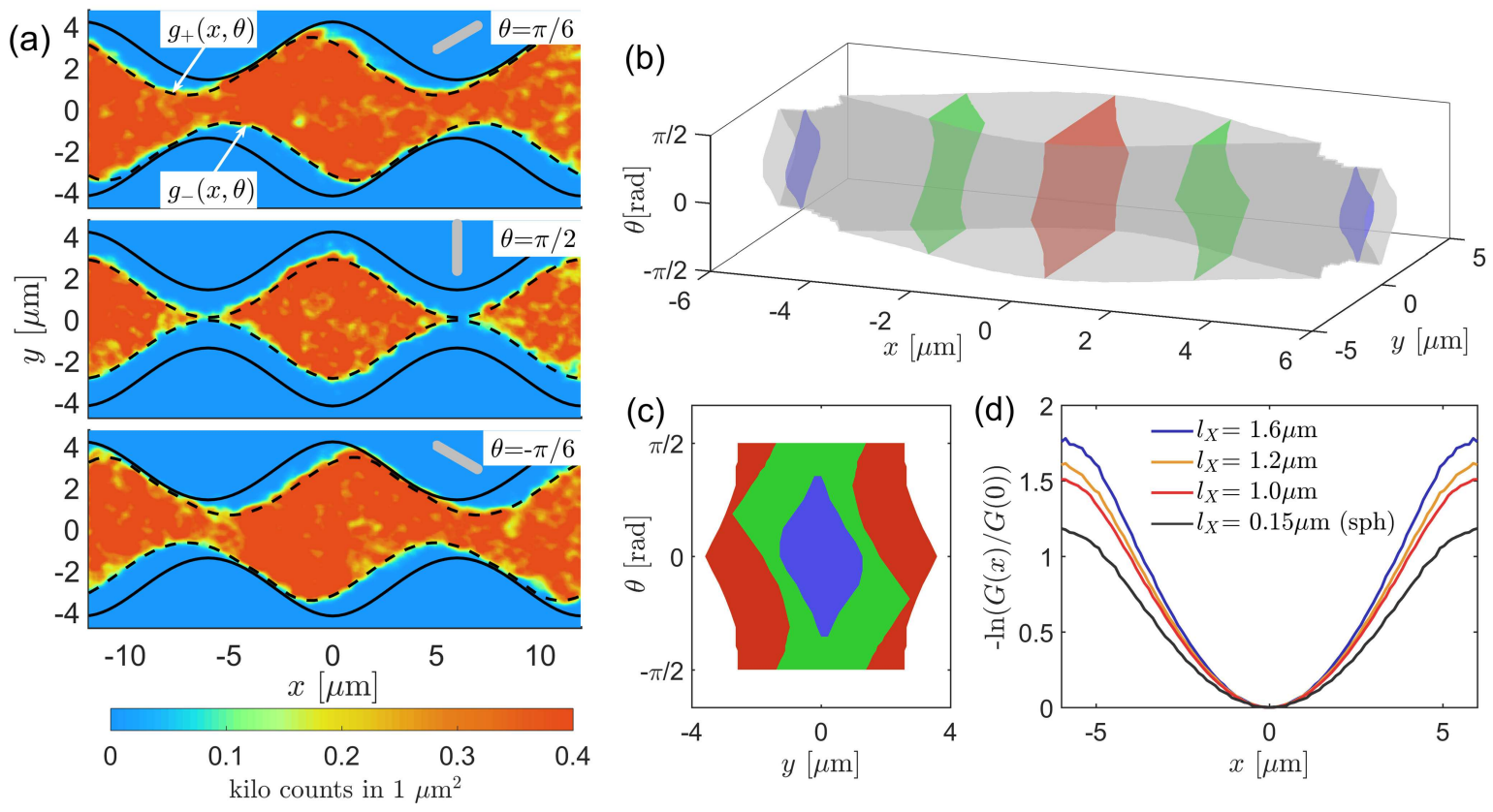

Figure 2. (a) Spatial distributions of the rod center for three tilting angles, $\theta=\frac{\pi}{6}, \frac{\pi}{2}$ and $-\frac{\pi}{6}$. The channel's inner boundaries, $y= \pm h(x)$, and the tilt-dependent effective boundaries, $y=g_{ \pm}(x, \theta)$, are marked by solid and dashed lines, respectively. (b) The configuration space accessible to the confined rod is delimited by the surfaces $y=g_{ \pm}(x, \theta)$. Five cross sections are shown in color; three of them, at $x / L=0,0.22$, and 0.46 , are displayed in (c). (d) Free-energy profile (in unit of $k_{B} T$ ), $-\ln [G(x) / G(0)]$, for different rod lengths (see text). The black line represents the case of a sphere of radius $l_{X}=0.15 \mu \mathrm{m}$. Data in (a)-(d) were obtained in a tall channel $(H=2.0 \mu \mathrm{m}, \alpha=1)$ with $h_{n}=1.8 \mu \mathrm{m}$, while the rod used in (a)-(c) had half-length $l_{X}=1.6 \mu \mathrm{m}$.

middle panel of Fig. 2(a).

Fick-Jacobs free-energy. The rod diffusion can be described as a random walk in the configuration space $(x, y, \theta)$. The dashed curves $y=g_{ \pm}(x, \theta)$ in Fig. 2(a) illustrate how the walls limit the channel's space accessible to the rod's center for three different $\theta$ values. From these curves one can construct a surface in the configuration space, as shown in Fig. 2(b), and model the motion of the confined rod as that of a pointlike particle diffusing inside the reconstructed 3D channel enclosed by that surface. For a rod with length of about $1 \mu \mathrm{m}$, the relaxation times of $\theta$ and $y$ are short enough for the FJ approach to closely reproduce the long-time diffusion in the reconstructed 3D channel (see supplemental Sec. IIB [36]). To that end, we integrate the probability density $\rho(x, y, \theta, t)$ to obtain $p(x, t)=\iint \rho(x, y, \theta, t) d y d \theta$ 
and the corresponding FJ equation governing its time evolution,

$$
\frac{\partial p(x, t)}{\partial t}=\frac{\partial}{\partial x}\left\{D(x)\left[\frac{\partial p(x, t)}{\partial x}+p(x, t) \frac{\partial}{\partial x}\left(-\ln \frac{G(x)}{G(0)}\right)\right]\right\} .
$$

Here, $G(x)=\frac{1}{2 \pi} \int_{-\pi / 2}^{\pi / 2}\left[g_{+}(x, \theta)-g_{-}(x, \theta)\right] d \theta$ represents the area of the $(y, \theta)$ cross section of the reconstructed $3 \mathrm{D}$ channel at a given point $x$. Three such cross sections are plotted in Fig. 2(c). Restrictions in both the center coordinates, $(x, y)$, and the tilting angle, $\theta$, cause variation of $G(x)$. The latter effect is most pronounced in the neck regions, as illustrated by the blue cross section in Fig. 2(c). Consequently, the variations of $G(x)$ modulate the FJ free-energy profile along the channel. The free-energy potentials plotted in Fig. 2(d), $-\ln [G(x) / G(0)]$, exhibit barriers of about $1.8 k_{B} T$ for a rod with a half-length $l_{X}=1.6 \mu \mathrm{m}$, which is $50 \%$ higher than that of a sphere. This novel entropic effect is induced by particle shape and its strength increases with increasing rod length.

Fick-Jacobs effective diffusivity. Apart from the entropic potential, the FJ approach introduces an effective longitudinal diffusivity function, $D(x)$ in Eq. 2. To estimate it, we first determined the local diffusivity matrix $\mathbb{D}_{I J}(x, y, \theta)$ of a rod located at $(x, y)$ with angle $\theta$, where $I$ and $J$ represent any pair of coordinates $X, Y$ or $\theta$ in the body frame. As shown in Fig. S1, off-diagonal elements of $\mathbb{D}_{I J}(x, y, \theta)$ are small and can be neglected. The remaining three diagonal elements, $\mathbb{D}_{X X}, \mathbb{D}_{Y Y}$ and $\mathbb{D}_{\theta \theta}$, exhibit a complicated structure inside the channel and generally have smaller values near channel boundaries, see Figs. S1(c)-(e). We also numerically computed the hydrodynamic friction coefficient matrix and then used the fluctuation-dissipation theorem to numerically estimate the diffusivity matrix. As shown with Fig. S3, numerical calculations closely reproduce experimental findings. Diffusivity at the channel center can be computed analytically [37-40] and results are in close (5\% difference) agreement with our findings.

We next transformed $\mathbb{D}_{I J}(x, y, \theta)$ from the body frame to the laboratory frame and then, in the spirit of the FJ theory, averaged the element of the resulting diffusivity matrix in the channel's direction, $\mathbb{D}_{x x}$, over $y$ and $\theta$ to obtain

$$
D_{\text {ave }}(x)=\left\langle\mathbb{D}_{x x}\right\rangle_{y, \theta}=\left\langle\mathbb{D}_{X X}(x, y, \theta) \cos ^{2} \theta+\mathbb{D}_{Y Y}(x, y, \theta) \sin ^{2} \theta-\mathbb{D}_{X Y}(x, y, \theta) \sin 2 \theta\right\rangle_{y, \theta}
$$

Figure 3(a) displays the function $D_{\text {ave }}(x)$ for three different rod lengths. While for the shortest $\operatorname{rod}\left(l_{X}=1.0 \mu \mathrm{m}\right) D_{\text {ave }}(x)$ exhibits minor variability along the channel, for the longest $\operatorname{rod}\left(l_{X}=1.6 \mu \mathrm{m}\right) D_{\text {ave }}(x)$ is about $30 \%$ larger in the neck regions than at the center 


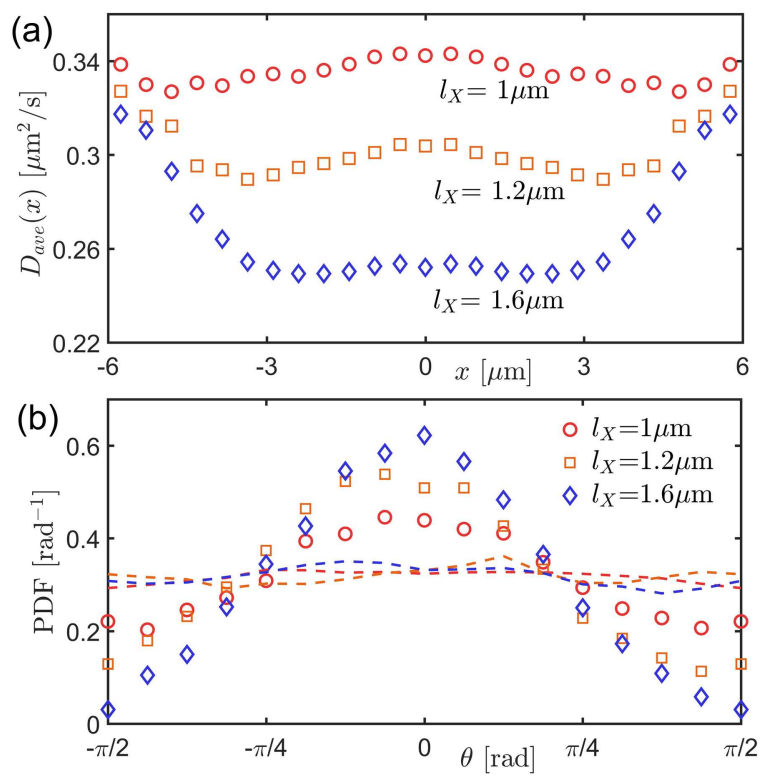

Figure 3. (a) Average local diffusivity, $D_{\text {ave }}(x)$, plotted along the channel for three rods with $l_{X}=$ 1, 1.2 and $1.6 \mu \mathrm{m}$, see corresponding numerical results in Fig. S3(c). (b) Tilting angle distributions in the neck regions $(x= \pm L / 2$, symbols), and at the center of channel cell $(x=0$, dashed lines), for the same $l_{X}$ as in (a). Data were taken in a tall channel $(H=2.0 \mu \mathrm{m}, \alpha=1)$ with $h_{n}=1.4$ $\mu \mathrm{m}$.

of the channel cells. This surprising result can be explained by inspecting the corresponding angular distributions in Fig. 3(b). While around the center of channel cell the rods can assume any angle, $\theta$, in the necks their orientation is predominantly constrained around $\theta=0$, more effectively as the rod length increases. In Eq. (3) for $D_{\text {ave }}(x)$, contributions of $\mathbb{D}_{X X}$ and $\mathbb{D}_{Y Y}$ are weighted respectively by $\cos ^{2} \theta$ and $\sin ^{2} \theta$, implying that for angular distributions peaked around $\theta=0$ the weight of $\mathbb{D}_{X X}$ becomes dominant. Moreover, Figs. S1 and S3 confirm that $\mathbb{D}_{X X} / \mathbb{D}_{Y Y} \approx 2$ in most of configuration space [41], so that $D_{\text {ave }}(x)$ in the neck regions is larger for longer rods. In addition to spatial variation, the hydrodynamic effects also cause a decrease of the local diffusivity of up to $25 \%$, as compared to bulk values (see Supplemental Sec. IIA [36]).

We next address the entropic corrections to the local diffusivity, $D_{\text {ave }}(x)$, which in the FJ scheme follow from the adiabatic elimination of the transverse coordinates [19, 20, 42]. Reguera and Rubí proposed heuristic expressions to relate $D(x)$ to $D_{\text {ave }}(x)$ in narrow $2 \mathrm{D}$ and 3D axisymmetric channels [20]. Unfortunately, such expressions do not apply to nonaxisym- 
metric "reconstructed" channels, see Fig. 2(b), where one or more d.o.f.'s are represented by orientation angles. For this reason we approximated the reconstructed 3D channel of Fig. 2(b) to a quasi-2D channel with half-width $G(x)$, adopted Reguera-Rubì expression [19, 20, 42] and arrived at the following estimate for $D(x)$,

$$
D(x)=\frac{D_{\text {ave }}(x)}{\left[1+G^{\prime}(x)^{2}\right]^{\frac{1}{3}}} .
$$

The validity and corresponding implications of Eq. (4) are discussed in Supplemental Sec. IIA [36].

Mean first-passage times. With both the entropic potential, $-\ln G(x) / G(0)$, and the effective logitudinal diffusivity, $D(x)$, as extracted from the experimental data, one can next apply the FJ equation to analytically study the diffusive dynamics of confined rods. For example, we focus on the time duration, $T( \pm \Delta x \mid 0)$, of the unconditional first passage events that start at $x=0$ and end up at $x= \pm \Delta x$ [see inset of Fig. 4(a)], regardless of the fastrelaxing coordinates $y$ and $\theta$. The corresponding MFPT, $\langle T( \pm \Delta x \mid 0)\rangle$, can then be used to estimate the asymptotic channel diffusivity in narrow-neck cases, i.e., $D_{\mathrm{ch}}=\lim _{t \rightarrow \infty}\langle[x(t)-$ $\left.x(0)]^{2}\right\rangle / 2 t$, that is $D_{\mathrm{ch}}=L^{2} / 2\langle T( \pm L \mid 0)\rangle[5]$. Taking advantage of the symmetry properties of the system, Eq. (3) returns an explicit integral expression for the MFPT [19, 43], reading:

$$
\left\langle T_{F J}( \pm \Delta x \mid 0)\right\rangle=\int_{0}^{\Delta x} \frac{d \eta}{G(\eta) D(\eta)} \int_{0}^{\eta} G(\xi) d \xi .
$$

In Fig. 4(a) we compare the predictions of Eq. (5) with the experimental measurements of $\langle T( \pm \Delta x \mid 0)\rangle$ for six combinations of $h_{n}$ and $l_{X}$. Without any adjustable parameters, Eq. (5) yields predictions in excellent agreement with the experimental data and captures the fast increase of the MFPT in the neck region. In addition, the validity of our generalized FJ equation has been systematically explored by extensive Brownian dynamics simulations in Supplemental Sec. ID [36].

Our experiments were controlled by two geometric parameters: the half-width of the channel's necks, $h_{n}$, and the rod half-length, $l_{X}$. Numerical and experimental results in Fig. 4 clearly reveal that the MFPT increases as the ratio $h_{n} / l_{X}$ decreases. Moreover, provided that the rods are not too short, $l_{X}>0.8 \mu \mathrm{m}$, results for different choices of $h_{n}$ and $l_{X}$, when plotted versus $h_{n} / l_{X}$, collapse onto a universal curve, as illustrated in Fig. 4(b). This means that, in the experimental regime investigated here, proportional increases of $h_{n}$ and $l_{X}$ do not 

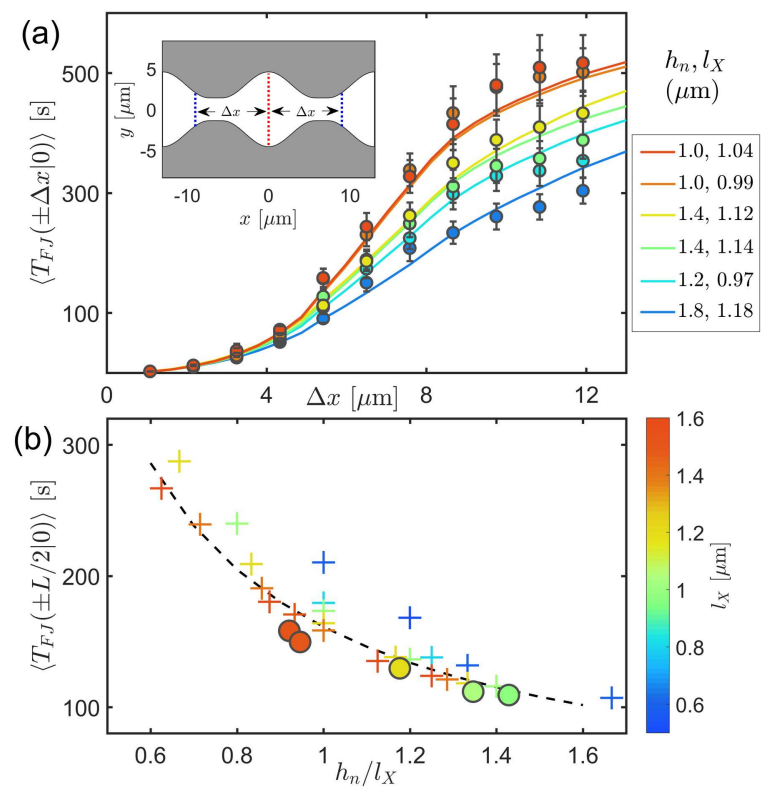

Figure 4. (a) MFPT $\langle T( \pm \Delta x \mid 0)\rangle$ vs. $\Delta x$ from experiments (symbols) and theory (curves) in thin channels $\left(H=1.0 \mu \mathrm{m}, \alpha=\frac{7}{8}\right)$ for different values of the pair $\left(h_{n}, l_{X}\right)$. Inset: vertical dashed segments mark the starting $(x=0$, red) and ending $(x= \pm \Delta x$, blue) positions of the recorded first passage events. (b) MFPT at $\Delta x=L / 2$ vs. $h_{n} / l_{X}$, measured in tall channels $(H=2.0 \mu \mathrm{m}$, $\alpha=1$ ) for different $h_{n}$ and $l_{X}$. Results from experiments and theory are represented by circles and crosses, respectively; symbols are color-coded according to the actual value of $l_{X}$. The local diffusivity, $D_{\text {ave }}(x)$, used in the theoretical computations was obtained via finite-element analysis, see Supplemental Sec. IIA [36]. The dashed line is a guide to the eye.

change the MFPT. For a qualitative explanation of such a property, we notice that increasing $l_{X}$ reduces the available configuration space and, simultaneously, raises the relevant entropic barriers [Fig. 2(d)]. As a consequence, longer rods, which also possess smaller diffusivity, $D(x)$ [Fig. 3(a)], tend to diffuse with longer MFPT's. On the other hand, increasing $h_{n}$ lowers the entropic barrier, thus decreasing the MFPT. As quantitatively discussed in Supplemental Sec. IIC [36], these two opposite effects tend to compensate each other, in our experimental regime, as long as the ratio $h_{n} / l_{X}$ is kept constant.

In conclusion, we experimentally measured diffusive transport of colloidal rods through corrugated planar channels, upon systematically varying the geometric parameters of the rods and the channel. Anisotropic shape significantly impacts particle transport by altering free-energy barriers and particle diffusivity. Experimental observations were successfully 
modeled by generalizing the FJ theory for spherical particles in terms of an effective longitudinal diffusivity, with hydrodynamic and entropic adjustments, and an FJ free energy including the rotational d.o.f.

Our method to quantify particle-shape-induced entropic effect (cf. Fig. (2)) is also applicable to model the confined diffusion of even more complex particles, like patchy colloids [28] or polymers [29, 30]. Such particles possess additional d.o.f.'s, other than the pure translational ones, and, similarly to the colloidal rods in our experiments, their description would generally require higher dimensional configuration spaces. However, as in our work, fast relaxing d.o.f.'s ("perpendicular" to the channel direction) may be adiabatically eliminated and replaced by a reduced free-energy potential [Fig. 2(d)] together with an effective diffusivity function [Eq. (4) and Fig. 3(a)]. Such a generalization of the FJ approach consequently may serve as a powerful phenomenological tool to accurately describe the diffusive transport of real-life particles in directed corrugated narrow channels.

Acknowledgments - We acknowledge financial supports of the NSFC (No. 11774222, 11422427, 11402069) and the Program for Professor of Special Appointment at Shanghai Institutions of Higher Learning.

[1] B. Berkowitz, A. Cortis, M. Dentz, and H. Scher, Rev. Geophys. 44, RG2003 (2006).

[2] M. J. Skaug, L. Wang, Y. F. Ding, and D. K. Schwartz, ACS Nano 9, 2148 (2015).

[3] C. Kettner, P. Reimann, P. Hänggi, and F. Muller, Phys. Rev. E 61, 312 (2000)

[4] S. Matthias and F. Müller, Nature 424, 53 (2003).

[5] X. Yang, C. Liu, Y. Li, F. Marchesoni, P. Hänggi, and H. P. Zhang, Proceedings of the National Academy of Sciences 114, 9564 (2017).

[6] M. J. Skaug, C. Schwemmer, S. Fringes, C. D. Rawlings, and A. W. Knoll, Science 359, 1505 (2018).

[7] F. Slanina, Phys. Rev. E 94, 042610 (2016).

[8] H.-X. Zhou, G. Rivas, and A. P. Minton, Annu. Rev. Biophys. 37, 375 (2008).

[9] P. C. Bressloff and J. M. Newby, Rev. Mod. Phys. 85, 135 (2013),

[10] P. Hänggi and F. Marchesoni, Rev. Mod. Phys. 81, 387 (2009). 
[11] P. S. Burada, P. Hänggi, F. Marchesoni, G. Schmid, and P. Talkner, ChemPhysChem 10, 45 (2009).

[12] O. Benichou and R. Voituriez, Phys. Rep. 539, 225 (2014).

[13] F. Hofling and T. Franosch, Rep. Prog. Phys. 76, 046602 (2013).

[14] W. M. Deen, AIChE J. 33, 1409 (1987).

[15] B. Hille, Ion Channels of Excitable Membranes (Sinauer Associates, 2001).

[16] J. Kärger and D. M. Ruthven, Diffusion in Zeolites and Other Microporous Solids (John Wiley, New York, 1992).

[17] M. Wanunu, T. Dadosh, V. Ray, J. M. Jin, L. McReynolds, and M. Drndic, Nat. Nanotechnol. 5, 807 (2010).

[18] M. Jacobs, Diffusion Processes (Springer, New York, 1967).

[19] R. Zwanzig, The Journal of Physical Chemistry, J. Phys. Chem. 96, 3926 (1992).

[20] D. Reguera and J. M. Rubí, Phys. Rev. E 64, 061106 (2001).

[21] P. Kalinay and J. K. Percus, Phys. Rev. E 74, 041203 (2006).

[22] D. Reguera, G. Schmid, P. S. Burada, J. M. Rubí, P. Reimann, and P. Hänggi, Phys. Rev. Lett. 96, 130603 (2006).

[23] A. M. Berezhkovskii, M. A. Pustovoit, and S. M. Bezrukov, J. Chem. Phys. 126, 134706 (2007),

[24] Y. Han, A. M. Alsayed, M. Nobili, J. Zhang, T. Lubensky, and A. Yodh, Science 314, 626 (2006).

[25] A. Chakrabarty, A. Konya, F. Wang, J. V. Selinger, K. Sun, and Q. H. Wei, Phys. Rev. Lett. 111, 160603 (2013).

[26] D. Kasimov, T. Admon, and Y. Roichman, Phys. Rev. E 93, 050602 (2016).

[27] F. Hofling, E. Frey, and T. Franosch, Phys. Rev. Lett. 101, 120605 (2008).

[28] S. Sacanna and D. J. Pine, Current Opinion in Colloid \& Interface Science 16, 96 (2011).

[29] N. Fakhri, F. C. MacKintosh, B. Lounis, L. Cognet, and M. Pasquali, Science 330, 1804 (2010).

[30] A. Ward, F. Hilitski, W. Schwenger, D. Welch, A. W. C. Lau, V. Vitelli, L. Mahadevan, and Z. Dogic, Nature Materials 14, 583 (2015).

[31] W. Reisner, K. J. Morton, R. Riehn, Y. M. Wang, Z. Yu, M. Rosen, J. C. Sturm, S. Y. Chou, E. Frey, and R. H. Austin, Phys. Rev. Lett. 94, 196101 (2005). 
[32] W. Riefler, G. Schmid, P. S. Burada, and P. Hänggi, Journal of Physics: Condensed Matter 22, 454109 (2010).

[33] C. Bechinger, R. Di Leonardo, H. Löwen, C. Reichhardt, G. Volpe, and G. Volpe, Rev. Mod. Phys. 88, 045006 (2016).

[34] C. Liu, C. Zhou, W. Wang, and H. Zhang, Phys. Rev. Lett. 117, 198001 (2016).

[35] J. C. Wu, Q. Chen, R. Wang, and B. Q. Ai, Chaos 25, 023114 (2015).

[36] See Supplemental Material for fabrication procedures, diffusion measurements, finite-element calculation, and Brownian dynamics simulations..

[37] J. Happel and H. Brenner, Low Reynolds Number Hydrodynamics (Prentice Hall, Englewood Cliffs, NJ, 1965).

[38] M. M. Tirado, C. L. Martínez, and J. G. de la Torre, The Journal of chemical physics 81, 2047 (1984).

[39] J. L. Bitter, Y. Yang, G. Duncan, H. Fairbrother, and M. A. Bevan, Langmuir 33, 9034 (2017).

[40] M. Lisicki, B. Cichocki, and E. Wajnryb, J. Chem. Phys. 145, 034904 (2016).

[41] Y. Han, A. Alsayed, M. Nobili, and A. G. Yodh, Phys. Rev. E 80, 011403 (2009).

[42] A. M. Berezhkovskii, L. Dagdug, and S. M. Bezrukov, J. Chem. Phys. 143, 164102 (2015).

[43] N. Goel and N. Richter-Dyn, Stochastic Models in Biology (Academic Press, 1974). 